\title{
Hemojuvelin is essential for dietary iron sensing, and its mutation leads to severe iron overload
}

\author{
Vera Niederkofler, Rishard Salie, and Silvia Arber \\ Biozentrum, Department of Cell Biology, University of Basel, and Friedrich Miescher Institute, Basel, Switzerland.
}

\begin{abstract}
Iron homeostasis plays a critical role in many physiological processes, notably synthesis of heme proteins. Dietary iron sensing and inflammation converge in the control of iron absorption and retention by regulating the expression of hepcidin, a regulator of the iron exporter ferroportin. Human mutations in the glycosylphosphatidylinositol-anchored protein hemojuvelin (HJV; also known as RGMc and HFE2) cause juvenile hemochromatosis, a severe iron overload disease, but the way in which HJV intersects with the iron regulatory network has been unclear. Here we show that, within the liver, mouse $H \mathrm{j} v$ is selectively expressed by periportal hepatocytes and also that $H$ jv-mutant mice exhibit iron overload as well as a dramatic decrease in hepcidin expression. Our findings define a key role for $\mathrm{Hj} v$ in dietary iron sensing and also reveal that cytokine-induced inflammation regulates hepcidin expression through an $\mathrm{Hj} v$-independent pathway.
\end{abstract}

\section{Introduction}

Regulation of iron uptake depends on the ability of an organism to accurately sense systemic iron and adjust its level accordingly. While iron is an essential physiological cofactor for the production of many proteins, most notably heme proteins, excess iron can be harmful to the organism, in part through the generation of oxygen radicals, and is potentially lethal $(1,2)$. Recent work has established the importance of the peptide hormone hepcidin in iron homeostasis as a negative regulator of iron release into the system by duodenal enterocytes and reticuloendothelial macrophages. Hepcidin binds to the iron exporter ferroportin, which results in ferroportin internalization and degradation (3). How hepcidin levels are kept in balance through upstream signaling pathways is still under investigation (4-7).

Multiple pathways are known to regulate expression of hepcidin and thus indirectly affect iron uptake and retention (7). Hepcidin expression is induced by an excess of iron and is downregulated by iron deprivation, consistent with its role as a downstream effector of iron sensing (8). In addition, hepcidin also responds to acute inflammation with rapid induction of gene expression. Injection of LPS, a bacterial endotoxin and potent activator of inflammatory response, induces hepcidin in mice (8), an effect believed to be dependent on cytokine production $(4,5)$. During inflammation induced by pathogenic infection, creation of a hypoferremic environment is thought to be a defense mechanism of the host organism for restricting pathogenic growth, which is partially dependent on physiological iron levels (9). Thus, both dietary iron sensing and inflammatory pathways converge in the regulation of the key regulator hepcidin, but how these 2 pathways intersect remains unclear.

An increasing number of genes have been assigned roles in iron homeostasis through their mutational identification in human

Nonstandard abbreviations used: ATG, amino-terminal methionine; eGFP, enhanced GFP; HJV, hemojuvelin; HNF4 $\alpha$, hepatocyte nuclear factor $4 \alpha$; RGM, repulsive guidance molecule; Tfr2, transferrin receptor 2 .

Conflict of interest: The authors have declared that no conflict of interest exists.

Citation for this article: J. Clin. Invest. 115:2180-2186 (2005).

doi:10.1172/JCI25683. diseases. Some of these mutations cause accumulation of iron and result in disease states of varying severity $(1,10)$. Human juvenile hemochromatosis is an early-onset disorder of iron homeostasis that results in massive iron overload in various body tissues (1, 10-12). Patients suffer from cardiomyopathy, diabetes, or cirrhosis attributed to oxidative damage caused by iron loading and often die before 30 years of age. Recently, a mutation causing juvenile hemochromatosis, which had been mapped to the human chromosomal position 1q, was identified by positional cloning (13). The corresponding gene was named hemojuvelin (HJV; also known as HFE2) and was shown to be a member of a previously identified glycosylphosphatidylinositol-anchored gene family named after its founding member, repulsive guidance molecule (RGM), the function of which has mainly been studied in the nervous system. The RGM gene family is composed of 3 members: $R G M a, R G M b$, and $R G M c$ (14). RGMc only differs from $H J V$ in nomenclature $(13,14)$, and its mouse homolog will be referred to as Hjv in this study. Whereas 2 mouse homologs of this family, mouse RGMa $(m R G M a)$ and $m R G M b$, are mainly expressed in the nervous system, the expression of Hjv is enriched in skeletal muscle and liver $(13,14)$. Functionally, $R G M a$ has been implicated in axon guidance and neural tube closure $(14,15)$ whereas the expression of $m R G M b$ (also known as DRAGON) is regulated by the transcription factor DRG11 in dorsal root ganglia sensory neurons (16). In contrast, no function for Hjv has previously been described.

Here we show that $H j v$ is expressed in periportal hepatocytes and that disruption of the Hjv gene results in severe iron overload. Mice with mutations in $H j v$ fail to express hepcidin in response to dietary or injected iron; this provides a molecular explanation for the severe iron accumulation observed in $\mathrm{Hj} v$-mutant mice. In contrast, these mice retain the ability to upregulate hepcidin expression in response to acute inflammation induced by either LPS or its downstream products IL- 6 and TNF- $\alpha$. Moreover, we also show that, upon induction of inflammation, Hjv expression in wild-type mice is selectively downregulated in the liver but not in skeletal muscle. Taken together with previous observations (6), our data suggest that inflammation, through downregulation of hepatic Hjv, might induce a temporary elimination of iron sensing. In summary, our 

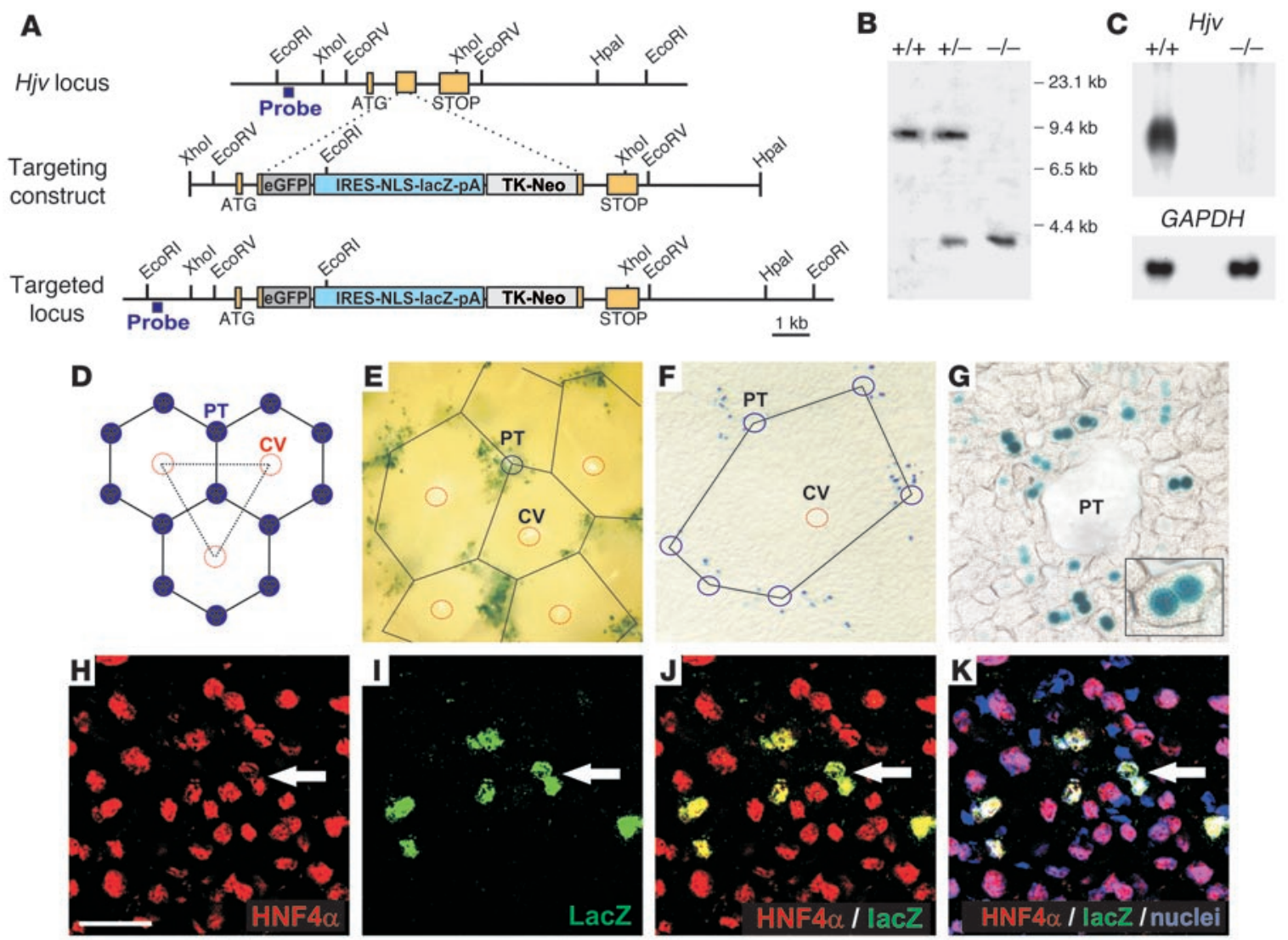

\section{Figure 1}

Hjv expression in periportal hepatocytes. (A) Targeting strategy used for homologous recombination in ES cells to eliminate Hjv gene function. The Hjv locus contains 3 coding exons (yellow). A targeting construct containing eGFP (dark gray) followed by IRES-NLS-lacZ-pA (blue) and thymidine kinase-neomycin (TK-neo) (light gray) cassettes was integrated in frame into the second coding exon of Hjv. The probe used for genomic Southern blot analysis is indicated in blue. Integrated cassette is not drawn to scale. STOP, carboxyterminal stop codon. (B) Genomic Southern blot of $\mathrm{Hjv}^{+/+}, \mathrm{Hjv}^{+/-}$, and $\mathrm{Hjv}^{-1-}$ genomic DNA using the probe indicated in A. (C) Northern blot analysis of total RNA isolated from P21 hindlimb muscles of $\mathrm{Hjv}^{+/+}$and $\mathrm{Hjv}^{-/-}$mice probed for the expression of $\mathrm{Hjv}$ (top) and GAPDH (bottom). (D) Schematic drawing depicting the territories of liver lobules. Portal tracts (PT) are indicated in blue; central veins (CVs) are shown in red. Note that solid lines in $\mathbf{D}-\mathbf{G}$ outline the hexagonally shaped hepatic lobule with PTs at the corners. $(\mathbf{E}-\mathbf{G})$ Detection of enzymatic lacZ activity in liver from 3-month-old $\mathrm{Hjv}^{+/-}$mice analyzed on vibratome $(\mathbf{E})$ or cryostat ( $\mathbf{F}$ and $\mathbf{G}$ ) sections. Red circles indicate $\mathbf{C V}$, blue circles indicate PT. Inset in (G) depicts high magnification of individual binuclear hepatocytes that express lacZ. (H-K) Immunohistochemical detection of HNF4 $\alpha$ (H, J, and K: red), lacZ (I, J, and K: green), and SYTOX green (nuclei; K: blue) in liver from 3-month-old $\mathrm{Hjv}^{+-}$mice. Arrows point to binuclear Hjv-expressing hepatocytes. Scale bar: 530 $\mu \mathrm{m}(\mathbf{E}) ; 260 \mu \mathrm{m}$ (F); $70 \mu \mathrm{m}(\mathbf{G}) ; 30 \mu \mathrm{m}$ (inset in $\mathbf{G}) ; 40 \mu \mathrm{m}(\mathbf{H}-\mathbf{K})$.

results suggest that Hjv plays an essential role in the regulation of bepcidin expression, specifically in the iron-sensing pathway.

\section{Results}

Generation of Hjv-mutant mice and expression of Hjv in periportal hepatocytes. The recent genetic linkage of $H J V$ to the iron overload disease juvenile hemochromatosis in humans (13) has opened the way to elucidation of the function of $\mathrm{Hj} v$ in iron homeostasis under normal physiological conditions and in disease by use of genetic studies in mice. We therefore generated $\mathrm{Hj} v$-mutant mice, which coordinately express lacZ targeted to the nucleus from the Hjv locus (Figure 1, $\mathrm{A}$ and $\mathrm{B})$. Previous work has shown that the strongest expression of Hjv in mice is found in skeletal muscles, but a lower level of expression has also been detected in the liver (14). Mice homozygous for the mutated Hjv allele showed a complete absence of Hjv mRNA in all tissues analyzed, including skeletal muscles, providing evidence for a complete null mutation (Figure 1C and data not shown).
To determine the exact site of expression of $H j v$ in the liver, we processed vibratome sections of adult liver from $\mathrm{Hjv}^{+/-}$mice in order to determine the presence of lacZ activity (Figure 1E). Interestingly, we found a patterned distribution of Hjv expression in the liver whereas skeletal muscles were stained uniformly (Figure 1E and data not shown). To determine the identity of these cells, we analyzed lacZ expression on thin sections and found labeled cells surrounding portal tracts but not central veins (Figure 1, F and G). At high magnification, lacZ ${ }^{+}$cells were often shown to contain 2 nuclei; this has previously been described as occurring frequently in hepatocytes $(17,18)$ (Figure 1G). In addition, double-labeling immunohistochemistry with an antibody against hepatocyte nuclear factor $4 \alpha$ (HNF4 $\alpha)$, a transcription factor expressed in hepatocytes (19), confirmed the hepatocytic identity of these lac $Z^{+}$ cells (Figure 1, H-K). In contrast, lacZ expression was not detected in any other of the several liver cell types examined, including sinusoidal endothelial cells expressing CD31 (20) (data not shown). 
Together, these findings indicate that Hjv expression in the liver is restricted to hepatocytes surrounding the portal tracts.

Hjv mutation in mice causes severe iron overload. To assess the consequences of $H j v$ mutation for iron homeostasis in various organs, we used both histological staining procedures and quantitative determination of iron content (Figure 2 and Supplemental Figure 1; supplemental material available online with this article; doi:10.1172/ JCI25683DS1). At 2.5 months of age, Hjv-mutant mice showed a severe increase in iron content in the liver ( $~ 20$ fold; iron accumulation in the parenchymal cells of the liver), pancreas ( 25 fold; iron accumulation in acinar tissue), and heart ( 4.5 fold) (Figure $2, A-D$, I, and J, and Supplemental Figure 1). In contrast, we found a reduction in iron accumulation in the spleen ( 4.5 fold; Figure $2 \mathrm{E}-\mathrm{H}, \mathrm{I}$, and J), probably due to the inability of reticuloendothelial macrophages residing in the red pulp to sequester iron. These findings are consistent with the previously observed distribution of iron content under conditions of hemochromatosis in both human patients and other mouse models of this disease $(10,11,21-24)$.

A time-course experiment for the determination of iron content in various tissues at several postnatal developmental stages of $\mathrm{Hj} v$ mutant mice showed a rapid and permanent increase in iron accumulation, which reached plateau levels by 4 months of age (Figure 2J and Supplemental Figure 1E). Importantly, the first signs of hepatic iron overload were already detected by P30 (Figure 2J). These findings reveal that mutation of $H j v$ in mice leads to iron accumulation in multiple organs with a time course and tissue distribution comparable to that observed in patients suffering from juvenile hemochromatosis (10).

Although we found similarities detected in iron accumulation between human juvenile hemochromatosis patients and Hjv-mutant mice, we did not observe obvious features of cardiomyopathy in these mice as assessed by histology, analysis of heart weight, and mRNA expression analysis of a number of marker genes known to be altered in cardiomyopathy (Supplemental Figure 1, F and G, and data not shown). Moreover, $\mathrm{Hjv}$ mutant mice did not experience an increase in mortality (up to 15 months of age) or show signs of diabetes (Supplemental Figure $1 \mathrm{H}$ and data not shown). While Hjvmutant males were sterile, they did not show signs of hypogonadism as assessed by determination of testicular size, a phenotype frequently observed in human sufferers of juvenile hemochromatosis (10). Together, these findings suggest that Hjv-mutant mice show an iron homeostasis phenotype highly similar to that of human patients but, surprisingly, do not develop all of the associated pathological conditions.

Lack of hepcidin expression in Hjv-mutant mice. We next began to assess the molecular mechanism by which absence of Hjv leads to iron accumulation in mice. Hepcidin expression is a well-established indicator of iron levels and is upregulated by high body iron (25). In the liver of wild-type rats, hepcidin expression occurs in 2 waves: an early postnatal spike (P0-P3) that declines rapidly followed by a second increase that begins during the fourth postnatal week and continues into adulthood (26). A very similar time course can be detected in mice in which adult levels of hepcidin expression in the liver are reached at P24 (6) (Figure 3A). In contrast to the dynamic expression of hepcidin, Hjv expression in the liver was already detected at E13.5 and reached a steady level by late embryonic stages (6) (Figure 3A).

We first determined the level of hepatic hepcidin expression in adult $H j v$-mutant mice by Northern blot analysis and in situ hybridization experiments. We found that bepcidin mRNA was virtually undetectable in adult $\mathrm{Hj} v$-mutant mice compared with wild-type littermates, in which expression was detected broadly throughout
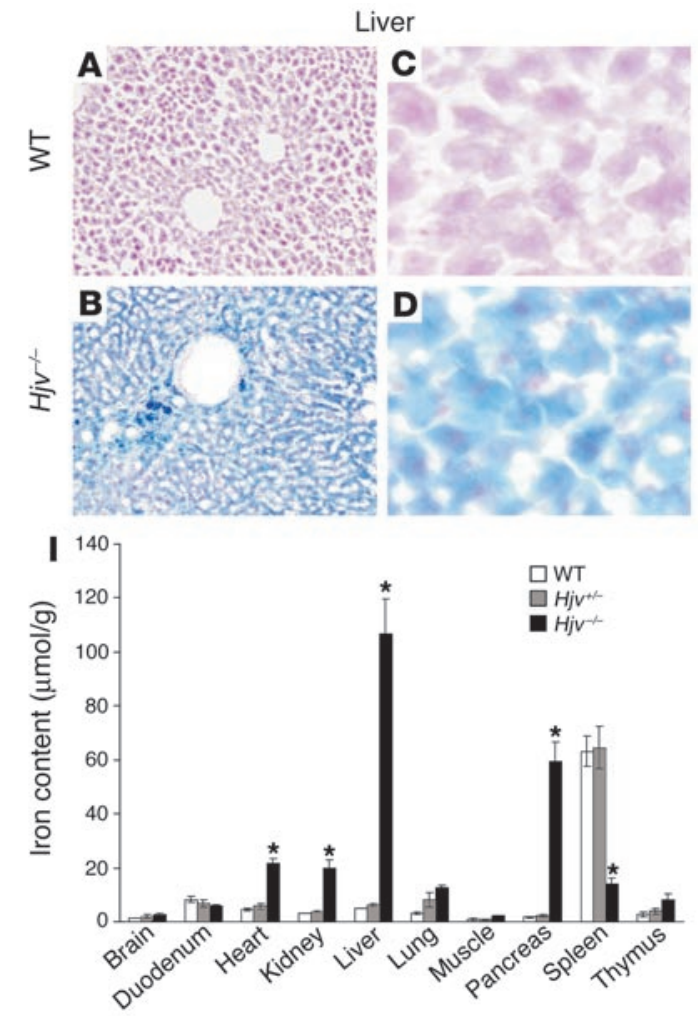

Figure 2

Iron accumulation in $\mathrm{Hjv}$-mutant mice. (A-H) Histological detection of iron content on cryostat sections of liver (A-D) and spleen (E-H) of wild-type (A, C, E, and $\mathbf{G})$ and $\mathrm{Hjv}^{-1-}(\mathbf{B}, \mathbf{D}, \mathbf{F}$, and $\mathbf{H})$ mice. Note uniform iron accumulation in the liver of 2.5-month-old Hjv-mutant mice and absence thereof in the red pulp of the spleen. (I) Quantitative determination of iron content ( $\mu \mathrm{mol} / \mathrm{g}$ dry weight) in various organs of 2.5-month-old wild-type (white), $\mathrm{Hjv}^{+/-}$(gray), and $\mathrm{Hjv}^{-1-}$ (black) mice ( $n=5$ for each group). Asterisks indicate significant changes $(P<0.05)$ in $\mathrm{Hjv}^{-1-}$ mice as compared with wild-type littermates. (J) Time course (P12-P300) of iron content ( $\mu \mathrm{mol} / \mathrm{g}$ dry weight) determined in $\mathrm{HjV}^{-1}$ mice (squares) compared with pooled wild-type and $\mathrm{Hjv}^{+-}$mice (triangles). Liver (green) and spleen (blue) are depicted in the graph. At least 3 animals per time point and genotype were included in the analysis. Asterisks indicate significant changes $(P<0.05)$ in $\mathrm{HjV}^{-/-}$mice as compared with pooled wild-type and $\mathrm{HjV}^{+/-}$littermates. Scale bar: $270 \mu \mathrm{m}$ (A and B); $45 \mu \mathrm{m}$ (C and $\mathbf{D}) ; 1.2 \mathrm{~mm}$ (E and F); $100 \mu \mathrm{m}$ (G and $\mathbf{H}$ ). 
A

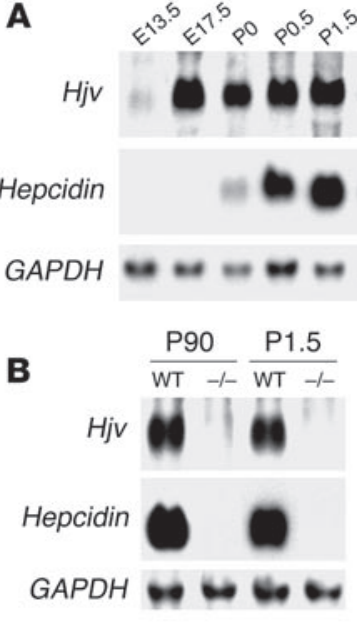

D

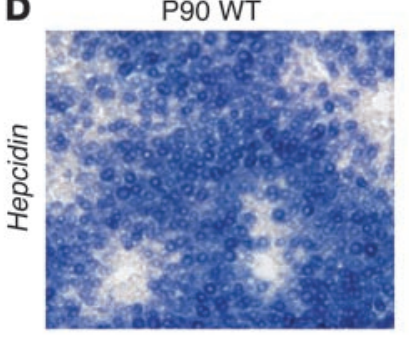

C

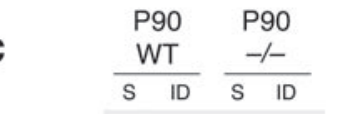

Hepcidin

GAPDH

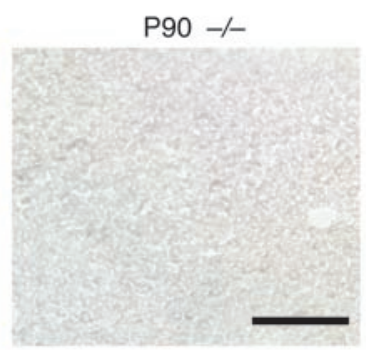

the liver ( $\leq 0.3 \%$ of wild type; Figure $3, \mathrm{~B}$ and D, and Figure $4 \mathrm{~A}$ ). Moreover, hepatic hepcidin expression in Hjv-mutant mice was also absent at early postnatal stages, when wild-type mice exhibit a naturally occurring spike of hepcidin expression (Figure $3 \mathrm{~B}$ ). To determine whether Hjv-mutant mice exhibit a general block of hepcidin regulation in response to iron, we assessed whether artificial elevation of iron levels in Hjv-mutant mice was capable of inducing hepcidin expression. We found that subcutaneous injection of irondextran (8) in Hjv-mutant mice did not increase hepcidin expression significantly whereas the same treatment consistently increased bepcidin in wild-type mice (Figure 3C).

Together, these findings point to an essential role for $\mathrm{Hj} v$ in iron sensing, that of an upstream regulator of hepcidin expression. Moreover, the massive reduction in hepcidin provides a molecular explanation for the continued iron accumulation and lack of effective regulatory mechanisms to decrease iron uptake in Hjv-mutant mice.

Acute inflammation can induce hepcidin expression in Hjv-mutant mice. Does the lack of hepcidin expression in Hjv-mutant mice represent an absolute inability to induce hepatic hepcidin expression, or is it possible to bypass this deficiency by stimulation of the inflammatory pathway $(4,8)$ ? We found that induction of acute inflammation by lipopolysaccharide (LPS) injection led to rapid and robust upregulation of hepcidin in Hjv-mutant mice compared with sham-injected mutant animals ( $\sim 300$ fold; Figure $4 \mathrm{~A})$. To determine whether downstream products of LPS were also sufficient to mimic the effect of LPS on hepcidin expression in $H j v$-mutant mice, we used injections of either proinflammatory cytokine IL- 6 or TNF- $\alpha$ (27). We found that either IL- 6 or TNF- $\alpha$ was sufficient to mimic the effect of LPS, albeit to a lesser extent (IL-6: 130 fold; TNF- $\alpha$ : 160 fold) (Figure 4A).

We also assessed whether, in Hjv-mutant mice, inflammationmediated upregulation of hepcidin expression was capable of effectively eliciting appropriate downstream responses. The iron

\section{Figure 3}

Lack of hepcidin expression in Hjv-mutant mice. (A) Developmental time course (E13.5-P90) of Hjv, hepcidin, and GAPDH expression levels as determined by Northern blot analysis on total RNA isolated from liver. (B) Northern blot analysis of total RNA isolated from adult (P90) or P1.5 liver of wild-type and $\mathrm{Hjv}^{-1-}$ mice probed for the expression of $\mathrm{Hjv}$, hepcidin, and GAPDH. (C) Northern blot analysis of total RNA isolated from adult (P90) liver of wild-type and $\mathrm{HjV}^{-1-}$ mice sacrificed 7 days after sham injection (S) or injection with iron-dextran (ID) and probed for the expression of hepcidin and GAPDH. Scale bar: $100 \mu \mathrm{m}$. (D) In situ hybridization on cryostat sections of liver isolated from adult (P90) wildtype and $\mathrm{Hjv}^{-/}$mice probed for the expression of hepcidin.

exporter ferroportin has been shown both to regulate cellular iron uptake by binding to hepcidin (3) and to be transcriptionally downregulated by high hepcidin levels (28). Consistent with the observed lack of hepcidin expression in Hjv-mutant mice, we found high expression of ferroportin in both untreated and sham-injected Hjv-mutant mice (Figure 4B and data not shown). In contrast, upon LPS injection (associated with hepcidin induction), ferroportin mRNA is significantly reduced in Hjv-mutant mice as well as in wild-type mice (Figure 4B), indicating the presence of intact downstream responses to hepcidin in Hjv-mutant mice. These findings show that the inflammatory pathway can efficiently bypass a requirement for $\mathrm{Hj} v$ in the induction of hepatic hepcidin expression and assign a specific role to Hjv in the iron-sensing pathway upstream of hepcidin regulation.

Inflammation induces selective downregulation of Hjv in liver but not muscle. Normal iron balance is subverted during inflammation when hepcidin levels are elevated to create a transient hypoferremic environment inhibitory to pathogenic growth (9). This low serum iron concentration should be perceived as hypoferremia by the dietary iron-sensing pathway and rapidly counteracted; however, this does not occur. Interestingly, previous experiments have shown that Hjv expression in the liver of wild-type mice is strongly downregulated upon induction of acute inflammation by LPS (6). These findings raise the question of whether the observed effect is selective to the liver and whether other genes involved in iron metabolism $(1,10-12)$ are regulated in a similar manner.

Interestingly, in contrast to the dramatic downregulation of $\mathrm{Hj} v$ expression observed in the liver of LPS-injected animals (6) (Figure 4C), no decrease in the expression of Hjv in skeletal muscles was detected under these conditions (Figure 4C). Moreover, we also found that the expression levels of several hemochromatosis- or iron metabolism-related genes, such as Hfe, transferrin receptor 2 (Tfr2), $\beta 2$-microglobulin, and ceruloplasmin, analyzed in the liver were unchanged following LPS injection (Supplemental Figure 2). Finally, a decrease in Hjv expression in the liver was also observed in response to IL-6 or TNF- $\alpha$ injection (Figure 4D). Together, these findings show that the inflammatory response induces a transcriptional downregulation of Hjv specifically in the liver and that such a response is not observed for other genes implicated in iron regulatory pathways.

\section{Discussion}

In this study, we provide evidence that Hjv expression in the liver is restricted to periportal hepatocytes and that Hjv is an essential component of the iron-sensing pathway. Our experiments show that Hjv-mutant mice exhibit an iron overload phenotype with high similarity to that of human patients suffering from juvenile hemochromatosis. Despite excessive iron accumulation, 

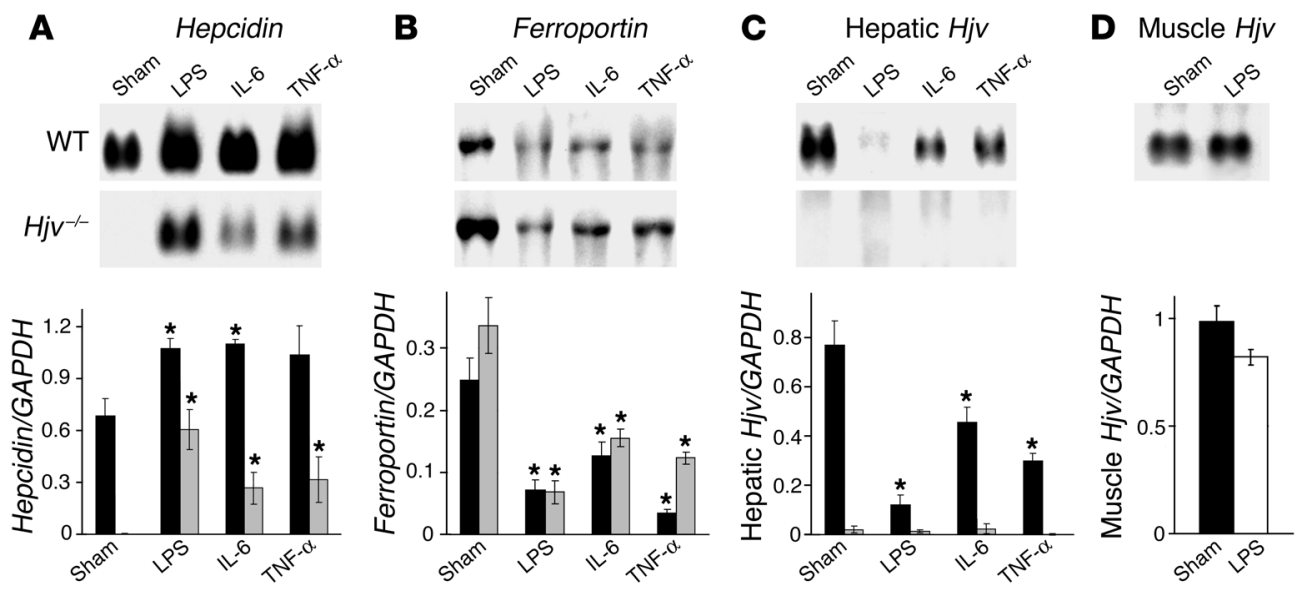

Figure 4

Selective suppression of Hjv during inflammatory response. (A-C) Northern blot analysis of hepcidin (A), ferroportin (B), and Hjv (C) expression on total RNA isolated from liver of wild-type or Hjv-mutant mice. Before isolation of total RNA, mice were injected intraperitoneally with PBS (sham), LPS, IL-6, or TNF- $\alpha$. At least 3 animals per experimental condition were analyzed, and 1 representative example is shown. Quantification of expression levels was performed by normalization of each sample to GAPDH expression probed sequentially on the same blots (data not shown). Histograms depict wild-type mice in black and Hjv-mutant mice in grey. Asterisks indicate significant changes $(P<0.05)$ in animals treated with LPS, IL-6, or TNF- $\alpha$ as compared with sham-injected animals of the same genotype. (D) Northern blot analysis of Hjv expression on total RNA isolated from skeletal muscle of wild-type mice after sham or LPS injection. Quantification was performed as described in (A-C). Histogram depicts sham-injected mice in black and LPS-injected mice in white.

Hjv-mutant mice show a complete lack of hepcidin expression; this provides a molecular explanation for the observed phenotype. Nevertheless, hepcidin expression can still be induced in Hjv-mutant mice by activation of the inflammatory pathway, providing evidence for the selective requirement of $\mathrm{Hj} v$ in the iron-sensing but not the inflammatory pathway upstream of hepcidin regulation. We will discuss our findings with respect to the role of Hjv in iron homeostasis and potential mechanisms by which Hjv might link iron sensing and inflammatory pathways in vivo.

The complex regulatory network underlying systemic regulation of iron homeostasis is tuned to respond to different stimuli by activation of distinct molecular pathways, all of which funnel into the regulation of hepatic hepcidin expression (7) (Figure 5, A and B). In this study, we provide evidence that $H j v$ is essential in the iron-sensing pathway. We found that even experimental elevation of iron levels was not capable of inducing hepcidin expression in $H j$-mutant mice and that these mice were also devoid of the hepcidin expression spike that normally occurs during the first postnatal week. These findings strongly suggest an essential role for $\mathrm{Hj} v$ in iron metabolism from birth throughout life.

In marked contrast to the defects in hepcidin expression in response to iron observed in Hjv-mutant mice, regulation of hep-

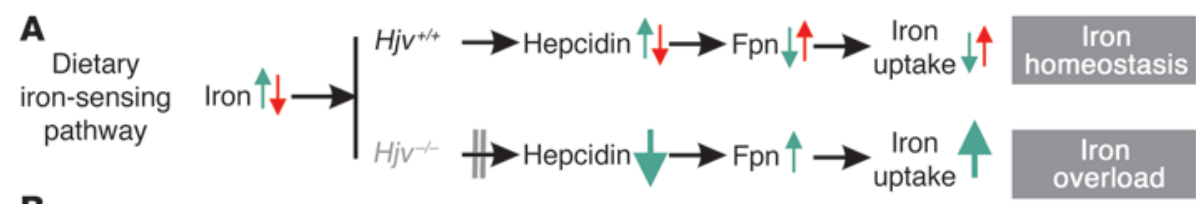

B

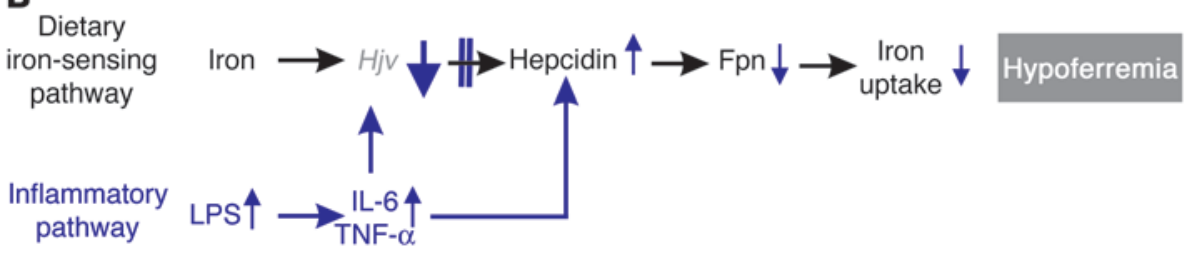

Figure 5

An essential role for Hjv in the iron-sensing pathway. (A) Model depicting the dietary iron-sensing pathway in wild-type and Hjv-mutant mice. In wild-type mice, green arrows indicate responses in the presence of high iron; red arrows show responses in the presence of low iron. Balanced regulation of this pathway adjusts iron levels to the needs of the healthy organism (follow green or red arrows from left to right). In Hjv-mutant mice, iron-sensing is defective due to the absence of $\mathrm{Hjv}$ (indicated by gray double line). Despite high iron, this leads to an essentially complete absence of hepcidin expression, iron overload, and hemochromatosis (green arrows in Hjv mutant). Fpn, ferroportin. (B) Model depicting the impact of acute inflammation on the iron-sensing pathway (blue arrows). Acute-phase cytokines IL- 6 and TNF- $\alpha$ act to coordinately downregulate Hjv expression in the liver while simultaneously inducing hepcidin expression. The reduction of $H j v$ results in a blockade of the dietary iron-sensing pathway (indicated by blue double line). This mechanism efficiently suppresses the iron-sensing pathway during the inflammatory response, which results in a low iron serum concentration inhibiting pathogenic growth. 
cidin expression upon inflammation is intact in these mutants. Interestingly, hepcidin levels in LPS-injected Hjv-mutant animals do not reach those of wild-type animals. These findings suggest that the total level of hepcidin expression observed upon inflammation is additive to the baseline level and again argue for the existence of 2 independent pathways that lead to the regulation of hepcidin expression. Of these 2 pathways, only the iron-sensing pathway requires functional $\mathrm{Hjv}$ (Figure 5).

How do iron sensing and inflammatory pathways interact, and what possible mechanism could prevent interference between the 2 pathways? Interestingly, previous experiments have shown that hepatic Hjv is rapidly downregulated at the transcriptional level upon induction of inflammation by LPS injection in wildtype mice (6). Moreover, we now show that Hjv downregulation upon inflammation is selective to the liver and does not occur in skeletal muscle, another prominent site of expression of Hjv (Figure 5B). Together with our observations that Hjv is not required for bepcidin induction during inflammation, these findings provide an intriguing potential mechanistic explanation for how the iron-sensing pathway is switched off during inflammation: by the rapid and selective extinction of $H j v$ in the liver (Figure 5B). By such a mechanism, interference with individual pathways may be prevented by selective and fast cross-regulatory interactions at the level of transcriptional gene regulation.

This study also provides evidence that the expression of Hjv in the liver is restricted to a population of periportal hepatocytes. In contrast, Hfe, Tfr2, and $\beta 2$-microglobulin have been described as expressed broadly throughout the liver (29-31). Periportal hepatocytes are located close to the portal veins, which deliver blood to the liver from the gut and are thus in a prime position to detect the iron content of blood coming directly from the digestive tract. While we cannot exclude a potential role for Hjv in ironsensing in skeletal muscles with the currently available mouse model, our results nevertheless suggest the possibility that the cellular source assigned to iron sensing in the liver may be periportal hepatocytes, marked by the expression of Hjv. Definitive proof of the importance of hepatic expression of $\mathrm{Hj} v$, however, awaits either the generation of tissue-specific $H j v$-mutant mice or attempts to selectively rescue $H j v$-mutant phenotypes by tissue-specific expression. Finally, since hepcidin expression is not restricted to periportal hepatocytes, this finding excludes a direct molecular link from $\mathrm{Hjv}$ to the intracellular induction of hepcidin expression.

In summary, the findings described in this study reveal a selective role for Hjv in 1 of 2 pathways, both of which converge on the downstream expression of the key regulatory peptide hepcidin. Whereas Hjv is required for hepcidin expression through the iron-sensing pathway, Hjv is dispensable for induction of hepcidin through the inflammatory pathway. Our findings also provide important insights for future therapeutic strategies for treating diseases affecting iron metabolism.

\section{Methods}

Generation, maintenance, and analysis of Hjv-mutant mice. A mouse genomic library was screened using an $H j v$-specific probe. The second coding exon of Hjv was disrupted via homologous recombination in ES cells (129SvJae1 origin; targeting frequency: $1: 100)$ by inserting a cassette containing an enhanced GFP (eGFP) in frame with the endogenous amino-terminal methionine (ATG), followed by an internal ribosomal entry site-nuclear localization signal-lacZ-polyA (IRES-NLS-lacZ-pA) and a thymidine kinase-neomycin. ES cell recombinants were screened by genomic Southern blot: EcoRI digest,
5' probe (300 bp); oligonucleotides, (a) 5'-CTCAGTGTATTATGTGTAGAA- $3^{\prime}$ and (b) 5'-AATTCCAGGAACGTTGGTGGC-3' (Figure 1A). The identification of $H j v$-mutant mice was performed by genomic Southern blot (Figure 1B) and PCR: (a) 5'-CCAGTGCAAGATCCTCCGCTGC-3' and (b) 5'-TCCGGATGGTGGTAGCGTTGGC-3'. Hjv mice were maintained in a $129 \mathrm{SvJ}$ genetic background under standard conditions. All experiments were performed using male mice, and control littermates were processed in parallel for each experiment. All animal experiments were approved by the Veterinarian Board Basel-Stadt (Basel, Switzerland).

Northern blot analysis and histology. Northern blot analysis and isolation of total RNA was performed as previously described (14), using digoxigeninlabeled probes directed against Hjv (14), hepcidin (BC021587), Tfr2 (BC013654), ß2-microglobulin (BI691504), Hfe (AA255260), ceruloplasmin (AI225600), ferroportin (BQ928442), and GAPDH (gift from P. Matthias, Friedrich Miescher Institute). Expression of $m M L P, \operatorname{Marp}, A N F(32), B N F$ (NM_008726), troponin1 (NM_009406), and MLC2a (33) were assessed by Northern blot analysis on heart total RNA. Signals were quantified using FluoView 500 (Olympus) and normalized to the expression level of GAPDH. Average values were determined from at least 3 independent experiments for each data point. Cryostat sections $(16 \mu \mathrm{m})$ were processed for immunohistochemistry as previously described (34), using fluorophore-conjugated secondary antibodies (1:1000; Invitrogen Corp.). Primary antibodies used in this study were rabbit anti-lacZ (34) and goat anti-HNF $4 \alpha$ (Santa Cruz Biotechnology Inc.). Nuclei were detected using SYTOX Green (Invitrogen Corp.). Vibratome sections (100 $\mu \mathrm{m})$ were cut on a vibratome (Leica). Detection of lacZ enzymatic activity and in situ hybridization experiments were performed as previously described $(14,34)$.

Iron quantification, blood glucose measurement, and statistical analysis. Iron was detected on cryostat sections using the Accustain Iron Stain Kit (SigmaAldrich). Nonheme iron in dehydrated tissue was quantified according to a previously described method (35). Animals were fasted for 6 hours before blood glucose measurement (Glucocard Memory 2, ARKRAY). For statistical analyses, all $P$ values were calculated with Microsoft Excel XP using a 2 -tailed Student's $t$ test. P values of less than 0.05 were considered statistically significant. Error bars shown in figures represent SEM.

LPS, cytokine, and iron injection. LPS ( $1 \mu \mathrm{g} / \mathrm{g}$ body weight; serotype O111: B4; Sigma-Aldrich), IL-6 (12.5 ng/g body weight; R\&D Systems), and TNF- $\alpha$ (12.5 ng/g body weight; R\&D Systems) were injected intraperitoneally, and organs were isolated for RNA preparation 6 hours after LPS and 4 hours after IL- 6 and TNF- $\alpha$ injections (28). Iron-dextran or PBS/dextran/phenolcontrol solution was injected subcutaneously as previously described, and animals were analyzed 7 days after injection (8).

\section{Acknowledgments}

We thank Jean-Francois Spetz, Patrick Kopp, Bernard Kuchemann, and Gisela Niklaus for excellent technical assistance; Nancy Andrews for sharing unpublished observations; and Caroline Arber, Pico Caroni, and Thomas Jessell for advice and helpful comments on the manuscript. S. Arber, V. Niederkofler, and R. Salie were supported by a grant from the Swiss National Science Foundation, the Novartis Research Foundation, and by the Kanton of Basel-Stadt.

Received for publication May 18, 2005, and accepted in revised form June 14, 2005.

Address correspondence to: Silvia Arber, Biozentrum, Department of Cell Biology, University of Basel, Klingelbergstrasse 70, 4056-Basel, Switzerland. Phone: 41-61-267-2057; Fax: 41-61-267-2078; E-mail: silvia.arber@unibas.ch.

Vera Niederkofler and Rishard Salie contributed equally to this work. 
1. Hentze, M.W., Muckenthaler, M.U., and Andrews, N.C. 2004. Balancing acts: molecular control of mammalian iron metabolism [review]. Cell. 117:285-297.

2. Andrews, N.C. 2005. Molecular control of iron metabolism. Best. Pract. Res. Clin. Haematol. 18:159-169.

3. Nemeth, E., et al. 2004. Hepcidin regulates cellular iron efflux by binding to ferroportin and inducing its internalization. Science. 306:2090-2093.

4. Nemeth, E., et al. 2004. IL-6 mediates hypoferremia of inflammation by inducing the synthesis of the iron regulatory hormone hepcidin. J. Clin. Invest. 113:1271-1276. doi:10.1172/JCI200420945.

5. Lee, P., Peng, H., Gelbart, T., and Beutler, E. 2004 The IL-6- and lipopolysaccharide-induced transcription of hepcidin in HFE-, transferrin receptor 2-, and beta 2-microglobulin-deficient hepatocytes. Proc. Natl. Acad. Sci. U. S. A. 101:9263-9265.

6. Krijt, J., Vokurka, M., Chang, K.T., and Necas, E. 2004. Expression of Rgmc, the murine ortholog of hemojuvelin gene, is modulated by development and inflammation, but not by iron status or erythropoietin. Blood. 104:4308-4310.

7. Nicolas, G., et al. 2002. The gene encoding the iron regulatory peptide hepcidin is regulated by anemia, hypoxia, and inflammation. J. Clin. Invest. 110:1037-1044. doi:10.1172/JCI200215686.

8. Pigeon, C., et al. 2001. A new mouse liver-specific gene, encoding a protein homologous to human antimicrobial peptide hepcidin, is overexpressed during iron overload. J. Biol. Chem. 276:7811-7819.

9. Luft, F.C. 2004. Hepcidin comes to the rescue. J. Mol. Med. 82:345-347.

10. Pietrangelo, A. 2004. Hereditary hemochromatosis-a new look at an old disease [review]. N. Engl. J. Med. 350:2383-2397.

11. Beutler, E., Hoffbrand, A.V., and Cook, J.D. 2003. Iron deficiency and overload [review]. Hematology. (Am. Soc. Hematol. Educ. Program.). 2003:40-61.

12. Brissot, P., Troadec, M.B., and Loreal, O. 2004. The clinical relevance of new insights in iron transport and metabolism. Curr. Hematol. Rep. 3:107-115.
13. Papanikolaou, G., et al. 2004. Mutations in HFE2 cause iron overload in chromosome 1q-linked juvenile hemochromatosis. Nat. Genet. 36:77-82.

14. Niederkofler, V., Salie, R., Sigrist, M., and Arber, S 2004. Repulsive guidance molecule (RGM) gene function is required for neural tube closure but not retinal topography in the mouse visual system. J. Neurosci. 24:808-818.

15. Monnier, P.P., et al. 2002. RGM is a repulsive guidance molecule for retinal axons. Nature. 419:392-395.

16. Samad, T.A., et al. 2004. DRAGON: a member of the repulsive guidance molecule-related family of neuronal- and muscle-expressed membrane proteins is regulated by DRG11 and has neuronal adhesive properties. J. Neurosci. 24:2027-2036.

17. Guidotti, J.E., et al. 2003. Liver cell polyploidization: a pivotal role for binuclear hepatocytes. J. Biol. Chem. 278:19095-19101.

18. Seglen, P.O. 1997. DNA ploidy and autophagic protein degradation as determinants of hepatocellular growth and survival. Cell Biol. Toxicol. 13:301-315.

19. Parviz, F., et al. 2003. Hepatocyte nuclear factor 4alpha controls the development of a hepatic epithelium and liver morphogenesis. Nat. Genet. 34:292-296.

20. Benten, D., et al. 2005. Hepatic targeting of transplanted liver sinusoidal endothelial cells in intact mice. Hepatology. doi:10.1002/hep.20746.

21. Kawabata, H., et al. 2005. Expression of hepcidin is down-regulated in TfR2 mutant mice manifesting a phenotype of hereditary hemochromatosis. Blood. 105:376-381

22. Fleming, R.E., et al. 2001. Mouse strain differences determine severity of iron accumulation in Hfe knockout model of hereditary hemochromatosis. Proc. Natl. Acad. Sci. U. S. A. 98:2707-2711.

23. Zhou, X.Y., et al. 1998. HFE gene knockout produces mouse model of hereditary hemochromatosis. Proc. Natl. Acad. Sci. U. S. A. 95:2492-2497.

24. Nicolas, G., et al. 2001. Lack of hepcidin gene expression and severe tissue iron overload in upstream stimulatory factor 2 (USF2) knockout mice. Proc. Natl. Acad. Sci. U. S. A. 98:8780-8785.

25. Andrews, N.C. 2004. Anemia of inflammation: the cytokine-hepcidin link. J. Clin. Invest. 113:1251-1253. doi:10.1172/JCI200421441.

26. Courselaud, B., et al. 2002. C/EBPalpha regulates hepatic transcription of hepcidin, an antimicrobial peptide and regulator of iron metabolism. Crosstalk between C/EBP pathway and iron metabolism. J. Biol. Chem. 277:41163-41170.

27. Zetterstrom, M., Sundgren-Andersson, A.K., Ostlund, P., and Bartfai, T. 1998. Delineation of the proinflammatory cytokine cascade in fever induction. Ann. N. Y. Acad. Sci. 856:48-52.

28. Yeh, K.Y., Yeh, M., and Glass, J. 2004. Hepcidin regulation of ferroportin 1 expression in the liver and intestine of the rat. Am. J. Physiol. Gastrointest. Liver Physiol. 286:G385-G394.

29. Chorney, M.J., Yoshida, Y., Meyer, P.N., Yoshida, M., and Gerhard, G.S. 2003. The enigmatic role of the hemochromatosis protein (HFE) in iron absorption. Trends Mol. Med. 9:118-125.

30. Zhang, A.S., Xiong, S., Tsukamoto, H., and Enns, C A 2004 . Localization of iron metabolismrelated mRNAs in rat liver indicate that HFE is expressed predominantly in hepatocytes. Blood. 103:1509-1514.

31. Kawabata, H., et al. 2001. Regulation of expression of murine transferrin receptor 2. Blood. 98:1949-1954.

32. Arber, S., et al. 1997. MLP-deficient mice exhibit a disruption of cardiac cytoarchitectural organization, dilated cardiomyopathy, and heart failure. Cell. 88:393-403.

33. Gottshall, K.R., et al. 1997. Ras-dependent pathways induce obstructive hypertrophy in echoselected transgenic mice. Proc. Natl. Acad. Sci.U.S. A. 94:4710-4715.

34. Arber, S., et al. 1999. Requirement for the homeobox gene Hb9 in the consolidation of motor neuron identity. Neuron. 23:659-674.

35. Torrance, J.D., and Bothwell, T.H. 1980. Tissue iron stores. In Methods in hematology. Volume 1. J.D. Cook, editor. Churchill Livingstone Press. New York, New York, USA. 90-115. 